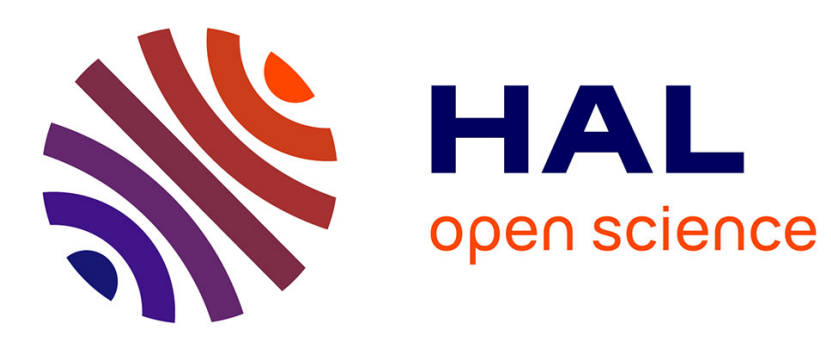

\title{
A rheological model for immersed corrugated elastic plates
}

Dominique Meier, Hervé Franklin, Mihai Valentin Predoi, Martine Rousseau, Jean-Louis Izbicki

\section{- To cite this version:}

Dominique Meier, Hervé Franklin, Mihai Valentin Predoi, Martine Rousseau, Jean-Louis Izbicki. A rheological model for immersed corrugated elastic plates. Ultrasonics, 2017, 75, pp.115-123. 10.1016/j.ultras.2016.11.018 . hal-01459715

\section{HAL Id: hal-01459715 https://hal.science/hal-01459715}

Submitted on 1 Sep 2021

HAL is a multi-disciplinary open access archive for the deposit and dissemination of scientific research documents, whether they are published or not. The documents may come from teaching and research institutions in France or abroad, or from public or private research centers.
L'archive ouverte pluridisciplinaire HAL, est destinée au dépôt et à la diffusion de documents scientifiques de niveau recherche, publiés ou non, émanant des établissements d'enseignement et de recherche français ou étrangers, des laboratoires publics ou privés. 


\title{
A rheological model for immersed corrugated elastic plates
}

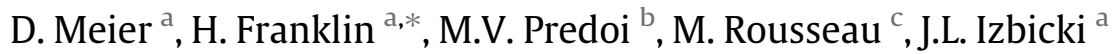 \\ a Laboratoire Ondes et Milieux Complexes, UMR 6294, Université du Havre, 75 rue Bellot, CS 80540,76058 Le Havre, France \\ ${ }^{\mathrm{b}}$ Department of Mechanics, University Politehnica Bucharest, Splaiul Independentei 313, Bucharest, Romania \\ ${ }^{\mathrm{c}}$ Institut Jean Le Rond d'Alembert, UPMC Université Paris 06, UMR 7190, F-75005 Paris, France
}

\begin{abstract}
The influence of surface imperfections on the propagation of guided waves in an immersed elastic plate can be interpreted by means of a rheological model. The corrugated surface is modeled by a very thin interface, similar to a Jones spring model, which replaces the continuity boundary conditions at the liquid - corrugated solid-plate interface. As the surrounding liquid is considered to be perfect, only one complex stiffness is used for the model of Jones. The selection of the plate guided mode and the test frequency are motivated by the detectability and non-interference with other modes. The spring stiffness is obtained by a best fit procedure, between the analytical solution and the results obtained by the finite elements method (FEM). One way ensuring the agreement of the two approaches, rheological and FEM, is to consider angular resonances provided by the transmission coefficients. Small changes in the parameters of the roughness keep the positions of the angular resonances of the plate practically unchanged, while at the same time large variations of the half width of the transmission coefficient curve is observed. The effect of corrugation parameters on the guided modes in the plate can be predicted by using the rheological model with the deduced spring complex stiffness.
\end{abstract}

\section{Introduction}

Machined plane surfaces are prone to imperfections, which can be described as local deviations from the theoretical plane surface. In most practical applications, the measurements are done along a selected direction in the plane, which can be assumed as the $O x$ axis. For a plate of average thickness $H$, the function $h_{r}(x)$ measured from the $H$ value, describes the surface imperfections. The Fourier spectrum of this function, can bring information about waviness (large periods) and roughness (short periods). In most cases, roughness is considered a random function, but experiments have shown that the spectrum can still exhibit several spatial periods. Roughness is in general defined by statistical functions such as $R_{q}$ [1], which is the root mean square (RMS) of the function $h_{r}(x)$ measuring deviations along a distance $x \in\left[0, L_{x}\right]$, in which $L_{x}$ is a characteristic length of the corrugation:

$R_{q}=\sqrt{\frac{1}{L_{x}} \int_{0}^{L_{x}} h_{r}^{2}(x) d x}$.

The geometrically imperfect interfaces between solids and liquids have raised a challenge in practical applications of ultrasonic wave

\footnotetext{
* Corresponding author.

E-mail address: herve.franklin@univ-lehavre.fr (H. Franklin).
}

propagation, for which both waviness and roughness should be taken into account. The earliest analytical treatment of a nonplanar reflection problem in acoustics was Rayleigh's solution (1893) for wave's reflection at a sinusoidal interface between two liquid media [2]. Miles investigated planar wave reflection at a rough interface between two liquids [3]. Biot considered a planar rigid wave reflector, for which the roughness is modeled by small hemispheres $[4,5]$. The reduction of the Rayleigh wave velocity due to roughness, which is considered as a uniform surface distribution of pyramids, has been theoretically deduced by Sinclair [6]. Measurements of scattered acoustic field by a random or periodic rough surface were performed by Quentin et al. [7] for water immersed blocks of aluminum at high frequencies (5-25 MHz), detecting diffraction peaks of the grating. Diffraction from water-acrylic saw-tooth interface, has been computed and measured by Chuang and Johnson [8] and for the opposite sense (solid-liquid) by Mampaert and Leroy [9]. The Rayleigh theory of reflection at rough surfaces with pressure release boundary conditions (total pressure cancels) has been investigated by Wirgin [10]. Reflection of ultrasonic pulses from rough rigid surfaces has been studied by Haines and Langston [11], introducing a roughness factor for planar surfaces. Ogilvy presents in his book [12] the most important theories on wave scattering from random rough surfaces, with emphasis on slightly rough surfaces for which the perturbation theories or Kirchhoff theories are widely 
applied. Numerical methods related to this problem are also presented. A critical survey of the analytical approximate methods that are encountered in scattering from random rough surfaces was performed by Elfouhaily and Guérin [13]. For a liquid-loaded flat plate, Fiorito, Madigosky and Überall (FMU) [14] deduced explicit mathematical expressions for the resonance amplitudes of transmission and reflection, based on series expansions about the free plate dispersion curves. The liquid loading effects are incorporated into the resonances widths, which can be related to the half-width of the angular resonances. In this way, computation of complex wavenumbers via an equation of dispersion is avoided. Resonances in the backscattered acoustic field of immersed plates were determined and measured by Maze et al. [15]. Pilarsky et al. [16] investigated the reflection and transmission coefficients of longitudinal and transverse waves travelling at oblique incidence in a tri-layer planar structure made of only solid materials. Vlasie and Rousseau [17] studied two plates bonded by an adhesive layer, using the same model, with and without spring mass.

Nagy and Adler $[18,19]$ investigated the attenuation of reflected and transmitted waves by a rough aluminum plate immersed in water, but only at normal incidence. Nagy extended the investigation on the various types of imperfect interfaces, by comparing several theories with experimental data. Duclos et al. [20] used the resonant formalism to describe Rayleigh's wave propagation at the liquid-solid interface. Their approach is similar to the one used in the present paper, but they focused on one perfectly plane interface, and only the Rayleigh wave was investigated. Drinkwater et al. [21] have investigated the reflection coefficient for normal incidence waves at the interface between two rough aluminum blocks. Their model integrates also a spring model, for which the normal stiffness $K_{L}$ is deduced as function of frequency and reflection coefficient. Chimenti and Lobkis [22] considered the plate thickness to be a complex number, with imaginary part accounting for the roughness $R_{q}$. The deduced modal attenuations for a wide frequency range were confirmed by experiments. The incident wave was on the smooth side of the plate and the corrugations on the opposite side of the water immersed plate.

In the present work, the Finite Element Method (FEM) is used to simulate the acoustic transmission through a plate with a periodic corrugation on only one face. The plate is immersed in water. The transmission coefficient is obtained as a function of the geometrical parameters of the corrugations. In the selected frequency range, the wavelengths in water are comparable with the period of the corrugation and diffraction effects are negligible at the positions of pressure evaluation, contrary to measurements in Ref. [7]. Depending on the wavelength, the spatial periodicity could induce additional effects such as band gaps or phonons [23]. These effects do not take place in the present study, because band gaps and phonons can be generated only when the ratio between the wavelength and the length of the periodic cell are small compared to unit. Thus, one deals with only the first Lamb modes of the plate, i.e., $S_{0}, A_{0}, S_{1}$ and $A_{1}$. The $A_{0}$ and $S_{0}$ modes are the only ones existing at all frequencies. Unfortunately, at fixed amplitude of excitation, due to a stronger attenuation, they do not propagate over a long distance in plates as $A_{1}$ and $S_{1}$ modes do. This is the reason for focusing on $A_{1}$ or $S_{1}$ modes. Since the analytical results for $S_{1}$ and $A_{1}$ do not differ qualitatively, we will emphasize on the computations for the $A_{1}$ mode.

The characteristics of the peaks of the transmission coefficients, such as width at half height, are obtained and interpreted within the classical model of resonances [14]. The frequency is chosen such that the two peaks are not overlapping. The attenuations of the leaky Lamb waves are then deduced by using the rheological model of Jones involving a complex spring stiffness. Our justification of the use of Jones model is the following: the corrugation being periodic, one can assume for small heights of the asperities with respect to the wavelength that the interface is composed of a very thin layer, neither liquid nor solid, separating liquid from plate. Furthermore, this layer is assumed to be homogeneous all along the plate and therefore can be modeled by using the rheological approach described by Jones.

The angles of the resonances are obtained from the complex valued solutions of the characteristic equation of the leaky Lamb waves. The main objective is to represent the attenuation of the leaky Lamb waves by a rheological model, with normal spring stiffness depending on the corrugation geometry, which can then provide much faster results than the FEM approach for practical applications.

\section{Theoretical background and extension}

We summarize in this section two approaches that will be used throughout the paper: the medialization of a corrugated surface by means of a rheological model and the concept of angular resonance which is a current alternative to the frequency resonances used for describing the characteristics of Lamb modes in plates.

\subsection{Rheological model}

Let us derive the boundary conditions at the interface between a viscous compressible liquid (index 1 ) and a perfectly plane elastic medium (index 2 ). It is well known that the constitutive equations for a viscous liquid can be written $(i, j=x, z)$

$\sigma_{1 i j}=-p_{1} \delta_{i j}+\tau_{1 i j}$,

where $\tau_{1 i j}=\lambda_{1} D_{1 k k} \delta_{i j}+2 \mu_{1} D_{1 i j}$ is the viscous stress tensor, with $D_{1 i j}=\left(\dot{u}_{1 i, j}+\dot{u}_{1 j, i}\right) / 2$ the tensor of the rate of deformation and $\delta_{i j}$ the second-order unit tensor and where $\lambda_{1}$ and $\mu_{1}$ are Lamé viscosity coefficients. The pressure $p_{1}$ depends on the liquid density. The notation $\dot{u}_{1 i}$ in $D_{1 i j}$ means time derivative of $u_{1 i}$ the component of the particle displacement vector. The stress tensor $\sigma_{2 i j}$ in the elastic medium depends on the strain tensor $\varepsilon_{2 i j}=\left(u_{2 i, j}+u_{2 j, i}\right) / 2$ through the relation

$\sigma_{2 i j}=\lambda_{2} \varepsilon_{2 k k} \delta_{i j}+2 \mu_{2} \varepsilon_{2 i j}$,

where $\lambda_{2}$ and $\mu_{2}$ are the Lamé elasticity coefficients. The boundary conditions at the viscous liquid- flat elastic interface are generally expressed by assuming the continuity of the displacement vector and that of the traction vector $\boldsymbol{T}=\boldsymbol{\sigma} . \boldsymbol{z}=\left(\sigma_{x z}, \sigma_{z z}\right)$ for the normal direction across the interface located at $z=+H / 2$, which mean:

$\left\{\begin{array}{l}u_{1 x}=u_{2 x}, \\ u_{1 z}=u_{2 z}, \\ \sigma_{1 x z}=\sigma_{2 x z}, \\ \sigma_{1 z z}=\sigma_{2 z z} .\end{array}\right.$

When the liquid is in contact with a corrugated surface, and in the hypothesis of acoustic waves with sufficiently large wavelengths incorporating several periods of corrugation, there exists a very thin region (located around the mean position $z=+H / 2$ in Fig. 1) which

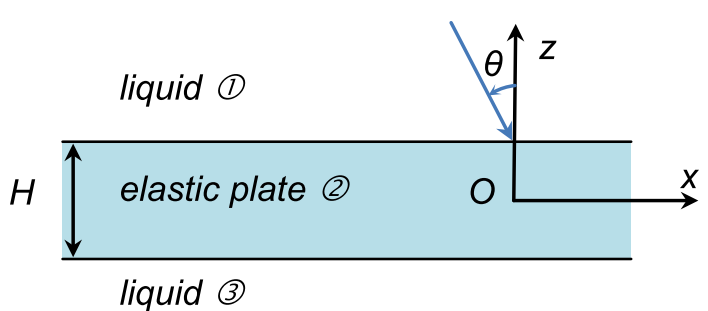

Fig. 1. The rheological model. 
presents complicated frictions between viscous liquid and elastic solid. A simple way of accounting for the effects of this interfacial region may be to consider the Voigt rheological model, for which a distribution of springs in parallel with viscous elements (dashpots) connects the displacements in the liquid and in the solid to the corresponding stresses. Similar to the Voigt model, Jones and Whittier [24] introduced a uniform distribution of longitudinal and shear springs, with respective complex stiffness, named $K_{L}$ in the normal direction $(\mathrm{Oz})$ and $K_{T}$ in the tangent direction (Ox).

Hence, we write the boundary conditions between the viscous liquid and the solid as:

$$
\left\{\begin{array}{cc}
K_{T}\left(u_{2 x}-u_{1 x}\right) & =\sigma_{2 x z}, \\
K_{L}\left(u_{2 z}-u_{1 z}\right) & =\sigma_{2 z z}, \\
\sigma_{1 x z} & =\sigma_{2 x z}, \\
\sigma_{1 z z} & =\sigma_{2 z z} .
\end{array}\right.
$$

In this set of equations, the components of the displacement and the components of the strain tensor are considered usually as functions of position and time [24] and the two stiffnesses of the springs are considered to be real valued. In order to solve the equation, a harmonic dependence on time $\exp (-i \omega t)$ is considered. In Ref. [17], a three-layer structure has been studied and the problem of two plates bonded by an adhesive layer was addressed with the same set of equations. Rheological models have been used to analyze the bonding: the stiffness coefficients of the springs are the parameters of the adhesion. When studying the cut-off frequencies of the modes propagating in the three-layered structure and the same structure with the rheological model, it has been shown that it is possible to obtain identical results by the two approaches, with stiffness depending on frequency. Strictly speaking, this evolution with the frequency, means that the position and time problem in (5) has to be substituted by a convolution problem and that the values for the spring stiffness are time dependent. As we are interested in guided waves, the set of Eq. (5) has to be understood as a Fourier transform of the time problem and consequently all the physical parameters shown in (5) are complex valued and functions both of position and frequency. In the following, the two stiffness values will be considered as complex parameters.

Next, the only way of agreeing with the case of a perfect liquid (i.e. $\lambda_{1}=\mu_{1}=0$ ) in slip contact with the corrugated interface, when the design of the considered roughness is "soft" or that anfractuosities of the roughness are not deep with slopes remaining small, is to assume that shear springs do not exist, i.e., $K_{T} \approx 0$. Consequently, the boundary conditions used for our computations reduce to

$$
\left\{\begin{array}{ccc}
K_{L}\left(u_{2 z}-u_{1 z}\right) & =\sigma_{2 z z}, \\
0 & =\sigma_{2 x z}, \\
-p_{1} & =\sigma_{2 z z} .
\end{array}\right.
$$

It is readily seen that when $\left|K_{L}\right| \rightarrow \infty$, the continuity of normal displacement $u_{2 z}=u_{1 z}$ is recovered. Thus, the boundary conditions for the corrugated interface become similar to those for a flat interface, a situation that occurs at $z=-H / 2$ where the boundary conditions can then be written

$$
\left\{\begin{array}{cl}
u_{3 z} & =u_{2 z}, \\
0 & =\sigma_{2 x z}, \\
-p_{3} & =\sigma_{2 z z} .
\end{array}\right.
$$

One recognizes the well-known slip boundary conditions at a liquid-solid interface.

\subsection{Angular resonances}

Let us assume a monochromatic plane wave $p_{\text {Inc. }}=p_{0} \exp \left[i\left(\boldsymbol{k}_{\text {Inc. }} \cdot \boldsymbol{r}-\omega t\right)\right]$

impinging onto the immersed plate, under of the incidence angle $\theta$, (Fig. 1). Here, $\boldsymbol{k}_{\text {Inc. }}=\left(k_{x}, k_{1 z}\right)=\left(k_{1} \sin \theta,-k_{1} \cos \theta\right)$ denotes the wave vector, $\boldsymbol{r}$ a position vector, $\omega$ the angular frequency, $t$ the time, $k_{1}=\omega / c_{1}$ the wavenumber in the liquid and $p_{0}$ the amplitude of the pressure. A dimensionless quantity is defined $\bar{k}_{x}=k_{x} / k_{1}=\sin \theta$, which will be used in subsequent sections. From the boundary conditions, Eqs. (6) and (7), one can find the expression of each of the two reflection coefficients $r_{1}$ and $r_{2}$, and that of the transmission coefficient $t_{1}$, such as given in Appendix A. In Eqs. (A1)-(A3) and subsequent, the index $a$ (s, resp.) indicates a quantity related to the antisymmetric (symmetric, resp.) modes of the flat plate. The coefficients $r_{1}, r_{2}$ and $t_{1}$ depend on the angular frequency $\omega$ and on the angle of incidence $\theta$. They depend on the corrugation, through the quantities $\boldsymbol{C}^{ \pm}$that tend to one as $\left|K_{L}\right|$ becomes infinite (flat plate limit).

Let us briefly present here the simplest cases of a free flat plate and of a flat plate loaded by a "light" liquid. The guided waves of the free flat plate, also called Lamb waves, are determined by solving the equations $C_{a}=0\left(C_{s}=0\right.$, resp.); each kind of wave ( $a$ or $s$ ) propagates independent from each other. The solutions of these equations are the dimensionless wave numbers $\bar{k}_{x}=\bar{k}_{x, p, a}$ (resp. $\bar{k}_{x}=\bar{k}_{x, p, s}$ ) which depend on the frequency, the integer $p$ being the order of the mode. When the flat plate is symmetrically loaded by a light liquid on the two faces, one speaks of "generalized" Lamb waves. When a "generalized" Lamb wave is generated, it is said that a resonance is excited. If $\theta$ is fixed whereas $\omega$ varies, the plot of the reflection coefficient $\left|r_{1 \text { flat }}\right|$ (the transmission coefficient $\left|t_{1 \text { flat }}\right|$, respectively) exhibits dips (peaks, respectively) called frequency resonances and characterized by both the values of the frequency at the maxima (minima, respectively) and the half-width [14]. If $\omega$ is held fixed whereas $\theta$ varies, dips in $\left|r_{1, f l a t}\right|$ (peaks in $\left|t_{1, f l a t}\right|$, respectively) describe angular resonances characterized by both the values of the angle at the maxima $\theta_{\text {flat }}^{*}$ and the half-width. In this last case, the perturbation brought to the solutions which were obtained in absence of liquid loading, is accounted for via an additional imaginary part, i.e., $\bar{k}_{x}=\bar{k}_{x, p, a}+\frac{i}{2} \gamma_{p, a}$ or $\bar{k}_{x}=\bar{k}_{x, p, s}+\frac{i}{2} \gamma_{p, s}$ where $\gamma_{p, a, s}$ is a real valued quantity defined below and $\bar{k}_{x, p, a, s}=\sin \theta_{\text {flat }}^{*}$.

Since the corrugation is small relative to the plate thickness, we will keep the same definition, i.e., "generalized" Lamb waves for the liquid loaded corrugated plate and examine only the angular resonances hereafter. Note that the presence of corrugation implies that $A$ and $S$ waves are normally coupled. This fact is explicit in Eqs. (A1)-(A3). But the expansion in resonant from obscures this point at the first order considered here. Therefore, the A and S modes continue to evolve independently from each other. When $\omega$ is held fixed whereas $\theta$ varies, dips appearing in $\left|r_{1}\right|$ or $\left|r_{2}\right|$ (peaks in $\left|t_{1}\right|$, respectively) describe angular resonances characterized by both the values of the angle at the maxima (minima, respectively) which do not change which respect to the flat plate, that is to say, $\theta^{*}=\theta_{\text {flat }}^{*}$ and the half-width. Let us assume, as in the Resonant Scattering Theory (FMU) [14] that the dips of interest in $\left|r_{1}\right|$ and $\left|r_{2}\right|$ (or the peaks in $\left|t_{1}\right|$ ) are well separated, that is to say, no overlapping occurs. At the neighborhood of an angle of resonance such that $\bar{k}_{x, p, a, s}=\sin \theta_{\text {flat }}^{*}$, the coefficients $r_{1}, r_{2}$ and $t_{1}$ can be expanded each in a form that borrows to the FMU theory, i.e.

$r_{1, p, a, s}=\frac{\boldsymbol{C}_{p, a, s}^{+}}{\boldsymbol{C}_{p, a, s}^{-}} \frac{\bar{k}_{x}-\bar{k}_{x, p, a, s}+\frac{i}{4}\left(\gamma_{p, a, s}^{+}-\gamma_{p, a, s}\right)}{\bar{k}_{x}-\bar{k}_{x, p, a, s}-\frac{i}{4}\left(\gamma_{p, a, s}^{-}+\gamma_{p, a, s}\right)}$,

$r_{2, p, a, s}=\frac{\bar{k}_{x}-\bar{k}_{x, p, a, s}-\frac{i}{4}\left(\gamma_{p, a, s}^{-}-\gamma_{p, a, s}\right)}{\bar{k}_{x}-\bar{k}_{x, p, a, s}-\frac{i}{4}\left(\gamma_{p, a, s}^{-}+\gamma_{p, a, s}\right)}$, 
$t_{1, p, a, s}=\frac{1}{\left(\mathbf{C}_{p, a, s}^{-}\right)^{1 / 2}} \frac{ \pm \frac{i}{2}\left(\gamma_{p, a, s} \gamma_{p, a, s}^{-}\right)^{1 / 2}}{\bar{k}_{x}-\bar{k}_{x, p, a, s}-\frac{i}{4}\left(\gamma_{p, a, s}+\gamma_{p, a, s}^{-}\right)}$,

where $\quad \gamma_{p, a}=-2 \tau_{p, a} / C_{a}^{\prime}\left(\bar{k}_{x, p, a}\right), \quad \gamma_{p, s}=2 \tau_{p, s} / C_{s}^{\prime}\left(\bar{k}_{x, p, s}\right)$, $\gamma_{p, a}^{ \pm}=-2 \tau_{p, a}^{ \pm} / C_{a}^{\prime}\left(\bar{k}_{x, p, a}\right), \quad \gamma_{p, s}^{ \pm}=2 \tau_{p, s}^{ \pm} / C_{s}^{\prime}\left(\bar{k}_{x, p, s}\right) \quad$ with $\tau_{p, a, s}=\tau\left(\bar{k}_{x, p, a, s}\right)$, $\tau_{p, a, s}^{ \pm}=\tau^{ \pm}\left(\bar{k}_{x, p, a, s}\right)$. The quantities $C_{a, s}^{\prime}\left(\bar{k}_{x, p, a, s}\right)$ are the values of the derivatives of $C_{a, s}$ with respect to the variable $\bar{k}_{x}$, considered at $\bar{k}_{x}=\bar{k}_{x, p, a, s}$. Here, $\boldsymbol{C}_{p, a, s}^{ \pm}=\boldsymbol{C}^{ \pm}\left(\bar{k}_{x, p, a, s}\right)$ with $\boldsymbol{C}^{ \pm}$defined in Appendix A. The quantity $\gamma_{\text {pa.s }}$ corresponds to the absence of roughness and the sum $\Gamma_{p, a, s}=\gamma_{p, a, s}+\gamma_{p, a, s}^{-}$to the width at half of the dips or peaks. It is also the width of $\left|t_{1}\right|$ at $-3 d B$ off the maximum. The quantities $\gamma_{p, a, s}$ and $\gamma_{p, a, s}^{ \pm}$are often called the partial widths of the resonances. It should be noted that $\Gamma_{p, a, s}$ specifies the state of each interface. In the absence of roughness, since $K_{L}$ is infinite, one obtains $\gamma_{p, a, s}^{ \pm}=\gamma_{p, a, s}$ from which it follows that $\left|r_{1, p, a, s}\right|=\left|r_{2, p, a, s}\right|=0$ and $\left|t_{1, p, a, s}\right|=1$.

Returning to Eqs. (9)-(11), it is straightforward that when $\bar{k}_{x}=\bar{k}_{x, p . a . s}$

$r_{1, p, a, s}=\frac{\boldsymbol{C}_{p, a, s}^{+}}{\boldsymbol{C}_{p, a, s}^{-}} \frac{\left(1-\boldsymbol{C}_{p, a, s}^{+}\right)}{\left(1+\boldsymbol{C}_{p, a, s}^{-}\right)}$,

$r_{2, p, a, s}=\frac{\left(1-\mathbf{C}_{p, a, s}^{-}\right)}{\left(1+\mathbf{C}_{p, a, s}^{-}\right)}$,

$t_{1, p, a, s}=\frac{2}{1+\mathbf{C}_{p, a, s}^{-}}$

The modulus of Eqs. (12)-(14) shows that a given resonance occurs with various strength in the three quantities $r_{1, p, a, s}, r_{2, p, a, s}$ and $t_{1, p, a, s}$.

\subsection{Analysis of the transmission coefficient}

Eqs. (11) may be written alternatively as

$t_{1, p, a, s}=\frac{ \pm \frac{i}{2} \gamma_{p, a, s} / \mathbf{C}_{p, a, s}^{-}}{\left[\bar{k}_{x}-\bar{k}_{x, p, a, s}-\frac{i}{2} \gamma_{p, a, s}\right]+\frac{i}{4} \gamma_{p, a, s}\left(1-1 / \mathbf{C}_{p, a, s}^{-}\right)}$,

where at the denominator, the term between the brackets [] corresponds to the flat plate and the remaining, to a perturbation brought to the case of the flat plate by the corrugated face. Let us consider the assumption of a complex valued quantity $K_{L}$ such as discussed above, i.e., $K_{L}=K_{L}^{\prime}(1+i \beta)$ where $\beta<1$. Then, from Eq. (A4),

$1-\frac{1}{\boldsymbol{C}_{p, a, s}^{-}} \approx-\beta \frac{K_{L 0, p, a, s}}{K_{L}^{\prime}}-i \frac{K_{L 0, p, a, s}}{K_{L}^{\prime}}$.

By substituting Eq. (16) in the denominator of the transmission coefficient while keeping the exact form of $\boldsymbol{C}_{p, a, s}^{-}$at the numerator, one obtains the rough approximation

$t_{1, p, a, s}=\frac{ \pm \frac{i}{2} \gamma_{p, a, s} / \mathbf{C}_{p, a, s}^{-}}{\left[\bar{k}_{x}-\bar{k}_{x, p, a, s}^{\prime}-\frac{i}{2} \tilde{\Gamma}_{p, a, s}\right]}$,

where

$$
\bar{k}_{x, p, a, s}^{\prime}=\bar{k}_{x, p, a, s}-\gamma_{p, a, s} K_{L 0, p, a, s} / 4 K_{L}^{\prime}
$$

$\tilde{\Gamma}_{p, a, s}=\gamma_{p, a, s}\left(1+\beta K_{L 0, p, a, s} / 2 K_{L}^{\prime}\right)$ are real valued quantities. The interpretation of Eq. (17) in the context of the resonance formalism is then that with respect to the flat plate, the rheological model adds: (a) a shift in position from $\bar{k}_{x, p, a, s}$ to $\bar{k}_{x, p, a, s}^{\prime}$, (b) a broadening of width from $\gamma_{p, a, s}$ to $\tilde{\Gamma}_{p, a, s}$ and (c) a diminishing of amplitude from 1 to $\left|\gamma_{p, a, s} / \mathbf{C}_{p, a, s}^{-} \tilde{\Gamma}_{p, a, s}\right|$. Eq. (17) will serve in the comparisons with the results obtained by FEM.
In the following, the transmission coefficients will be analyzed for a corrugated plate and the resonances will be characterized thanks to two ratios:

$\eta_{\theta}=\frac{\bar{k}_{x, p, a, s}-\bar{k}_{x, p, a, s}^{\prime}}{\bar{k}_{x, p, a, s}}=\frac{-\gamma_{p, a, s}}{4 \bar{k}_{x, p, a, s}} \frac{K_{L 0, p, a, s}}{K_{L}^{\prime}}$

and

$\eta_{\gamma}=\frac{\gamma_{p, a, s}-\tilde{\Gamma}_{p, a, s}}{\gamma_{p, a, s}}=\frac{-\beta}{2} \frac{K_{L 0, p, a, s}}{K_{L}^{\prime}}$,

which measure the sensitivity of the angular position and of the resonance width, respectively, to various surface corrugation parameters. The values of $\eta_{\theta}$ and $\eta_{\gamma}$ will be computed at first with FEM and then used to obtain the values of $K_{L}^{\prime}$ and $\beta$. This aspect is developed below.

\section{Simulation of the acoustic scattering by an immersed corrugated plate}

The corrugation is assumed periodic and the repeated corrugation geometry is triangular [12]. In fact, real surface roughness obtained from various machining technologies, can be measured with a profilometer and the "power spectrum" of the profilometer measurements proves in most cases that the roughness is not randomly distributed and moreover, only one or a few wavelengths are dominant [23]. Basically, this can be assumed as a first harmonic approximation. The RMS derived from Eq. (1) for the function

$h_{r}(x)=\left\{\begin{array}{cc}(-x / a+1) h_{r} & 0<x<a \\ (x / a+1) h_{r} & -a<x<0\end{array}\right.$

is

$R_{q}=h_{r} \sqrt{\frac{2 a}{3 L_{x}}}$,

where $2 a / L_{x}$ is defined as the filling fraction. The height $h_{r}$ (Fig. 2a) of the triangular corrugation is considered as one of the parameters, and lies between 0 and $0.2 \mathrm{~mm}$, which is very small compared to the average thickness $(H=5 \mathrm{~mm})$ of the plate.

Thus, the corrugation is a small disturbance as defined in previous studies [25,26]. The length of the periodic cell is $L_{x}=8 \mathrm{~mm}$, a value comparable with the wavelength of the Lamb wave propagating in the flat plate in the selected frequency range. Thus no band gaps or similar effects are to be expected. The base of the triangle $2 a=6 \mathrm{~mm}$ (both values of $L_{x}$ and $a$ given here are for the reference case). A two-dimensional plane strain problem is considered. The plate is made of stainless steel, of density $\rho_{s}=7800 \mathrm{~kg} / \mathrm{m}^{3}$, longitudinal and transversal wave speeds $c_{L}=6020 \mathrm{~m} / \mathrm{s}$ and $c_{T}=3220 \mathrm{~m} / \mathrm{s}$ respectively. Water density and speed of sound are $1000 \mathrm{~kg} / \mathrm{m}^{3}$ and $c_{1}=1500 \mathrm{~m} / \mathrm{s}$, respectively. For all corrugations $\left(h_{r} \neq 0\right)$, the average thickness of the plate, determined as $H=h_{p}-a h_{r} / L_{x}$, is kept constant as the other parameters of the corrugation are changed. This is consistent with the definition of roughness parameters relative to the average thickness, as indicated in the introduction.

\subsection{Dispersion curves and resonance peaks for the immersed flat plate}

A harmonic progressive plane wave similar to that given by Eq. (9) impinges the plate on the corrugation side under the incidence angle $\theta$ as shown on Fig. 1. For the immersed flat plate, the cancellation of the denominator of the reflection or transmission coefficients yields the dispersion equations $C_{a}+i \tau=0$ and $C_{s}-i \tau=0$. 


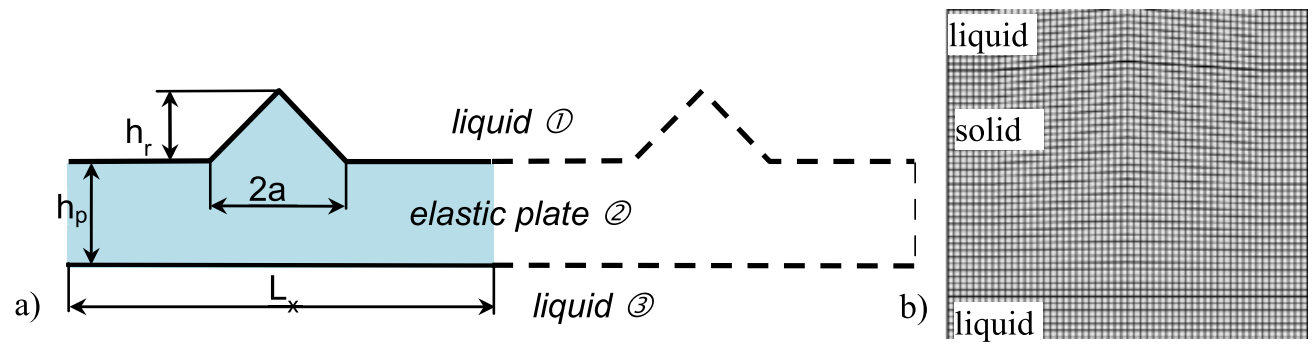

Fig. 2. Geometry of the immersed corrugated plate (a) and FEM mesh detail (b).

The solutions of physical interest $\bar{k}_{x}(\omega)$ of these equations are complex in general and can be written

$\bar{k}_{x}=k_{x}^{\prime}+i k_{x}^{\prime \prime}$,

in which both $k_{x}^{\prime}$ and $k_{x}^{\prime \prime}$ are real valued. The link with the angle of incidence $\theta$ and the width $\gamma$ introduced in Section 2 is achieved by setting:

$k_{x}^{\prime}=\sin \theta_{\text {flat }}^{*}=\bar{k}_{x, p, a, s}, \quad k_{x}^{\prime \prime}=\frac{1}{2} \gamma_{p, a, s}$.

The curves of $\sin \theta$ are shown in Fig. 3a versus the frequency in the range $60-900 \mathrm{kHz}$ where the symmetric modes $S_{0}, S_{1}, S_{2}$ as well as the anti-symmetric modes $A_{0}, A_{1}$ exist. For a fixed frequency, as the angle of incidence $\theta$ crosses a particular value $\theta_{\text {flat }}^{*}$, the transmission coefficient of the plate exhibits one peak of total transmission (its maximum amplitude, which is equal to one, is reached for $\theta=\theta_{\text {flat }}^{*}$ ). For example in Fig. $3 \mathrm{~b}$, the modulus of the transmission coefficient $t_{1}$ when plotted versus $\sin \theta$ at the frequency $f=600 \mathrm{kHz}$, shows that four different values $\theta_{\text {flat }}^{*}$ have been crossed, allowing the identification of the four guided Lamb modes: $S_{1}$ $\left(\bar{k}_{x, p, s}=0.216\right), \quad \mathrm{A}_{1} \quad\left(\bar{k}_{x, p, a}=0.240\right), \quad \mathrm{S}_{0} \quad\left(\bar{k}_{x, p, s}=0.436\right) \quad$ and $\quad \mathrm{A}_{0}$ $\left(\bar{k}_{x, p, a}=0.521\right)$. An optimal frequency range (around $600 \mathrm{kHz}$ in this case) can be determined for any plate, requiring that the two transmission peaks of modes $A_{1}$ and $S_{1}$ are well separated.

\subsection{Behavior of the resonance peaks of the corrugated plate using FEM}

The numerical study, in presence of small corrugations such as those considered here, is carried out using rectangular finite elements implemented in a commercially available software [27].
The possible choice of triangular finite elements has no influence on the computation accuracy, but the computation time increases.

A FEM mesh on the bounded domain of interest (a unit cell of length $L_{x}$ ) is shown in Fig. 2b. The maximum size of rectangle sides is $0.2 \mathrm{~mm}$. It is considered that the total pressure $p(x, z)=p_{i}(x, z)$ $+p_{s}(x, z)$ is periodic: $p(0, z)=p\left(L_{x}, z\right) e^{i k_{x} L_{x}}$, in the two liquid subdomains, in which $p_{i}$ and $p_{s}$ are the incident (null on the lower liquid domain) and reflected/transmitted pressure respectively.

The same periodicity is imposed on the displacement fields for the elastic sub-domain. It is assumed that this periodicity, which is exact in the absence of corrugation, remains valid in the case of small geometrical perturbations. The boundary conditions applied at each liquid-elastic body interfaces are (i) continuity of normal accelerations, (ii) continuity between pressure and normal component of traction. The two liquid domains are normally of infinite extent. In order to avoid spurious reflections, the fluid domains are extended by two Perfectly Matched Layers (PML) [28].

In order to ensure consistent assessment of the viability of the FEM, one considers at first a flat plate having the characteristics described at paragraph 3 . All the results obtained with the FEM model agree very well with those presented in Fig. 3(a) and (b). The procedure is then applied to the plate with a corrugated surface. Small amplitude diffracted waves on the corrugated side have been determined; but developments about this aspect is not the purpose of this paper. For the selected small corrugations, the energy balance is verified with $2.5 \%$ maximum error. Moreover, we use the transmitted pressure field in all computations, which is less affected by the diffraction produced on the insonified side.

It has been seen, Eq. (21), that the three parameters $h_{r}, a$ and $L_{x}$ are contained in the RMS $R_{q}$. Their influences on the transmission peak of the anti-symmetric mode $A_{1}$ are presented below. The values of reference are $h=0 \mathrm{~mm}, a=3 \mathrm{~mm}$ and $L_{x}=8 \mathrm{~mm}$. Four cases
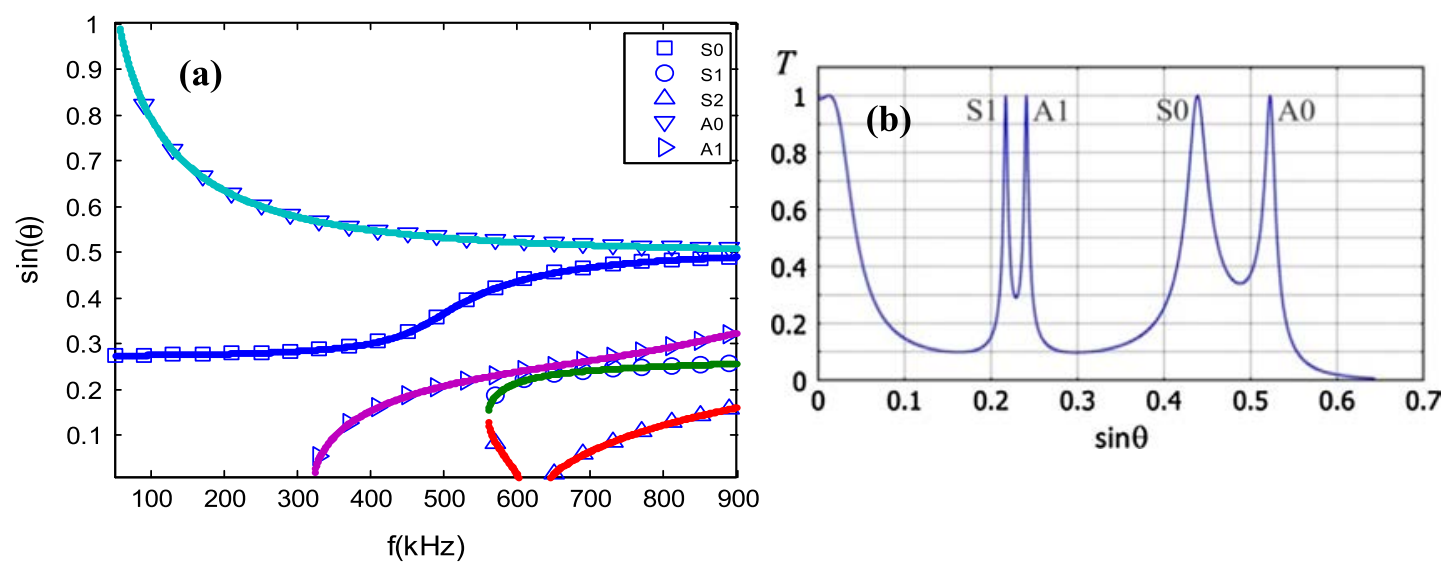

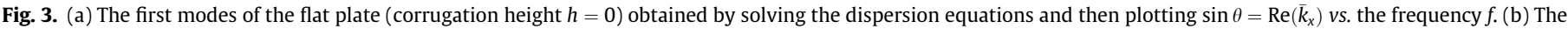
four first modes at the frequency $f=600 \mathrm{kHz}$ obtained by plotting the modulus of the transmission coefficient $\left|t_{1}\right| v s$. sin $\theta$. 
are successively analyzed. In the first three cases, the RMS represented by Eq. (21) increases. In the last case, the RMS is kept constant.

\subsubsection{Effect of the corrugation height}

The triangle height $h_{r}$ increases $\left(a=3 \mathrm{~mm}\right.$ and $L_{x}=8 \mathrm{~mm}$ are fixed) from 0 to $0.2 \mathrm{~mm}$, in agreement with the assumption of small corrugations. As shown in Fig. 4(a) for the $A_{1}$ mode at $600 \mathrm{kHz}$, both the amplitude and the width of the peak of the transmission coefficient are modified. The shift of the peak to the left indicates that the angle corresponding to the maximum in transmission, diminishes with the corrugation height. The diminishing of the amplitude is monotonic as $h_{r}$ increases. The curve being smooth, it is not difficult to compute the width of the peaks at $-3 \mathrm{~dB}$ of the maximum. The influence of an increase of $h_{r}$ on the width is however weak.

\subsubsection{Effect of triangle size vs. length of unit cell}

In this case, is the base $2 a$ of the triangle which increases ( $h_{r}=0.12 \mathrm{~mm}$ and $L_{x}=8 \mathrm{~mm}$ are fixed), meaning that the corrugation occupies more and more space of the $8 \mathrm{~mm}$ width cell. In Fig. 4 (b), the transmission coefficient is presented for several values of $a$ ranging from $0 \mathrm{~mm}$ (plane plate) to $3.6 \mathrm{~mm}$. As in Section 3.2.1, the peak of the transmission coefficient shifts towards lower angles. Thus, the increase of the $R_{q}$, which results from the variations of the parameters $a$, leads to an evolution of the transmission coefficient similar to Fig. 4(a).

The increasing occupation of the unit cell by the triangle, influences only slightly the amplitude and the width of the Lamb mode $A_{1}$, in comparison with influence of height of the corrugation.

\subsubsection{Effect of the distance between triangles}

In this case, the width $L_{x}$ of the unit cell of the corrugation decreases ( $h_{r}=0.12 \mathrm{~mm}$ and $a=3 \mathrm{~mm}$ are fixed). In Fig. 4(c), are shown the effects of a decrease of $L_{x}$ from $11 \mathrm{~mm}$ to $0 \mathrm{~mm}$ (this last value, because of the constraint $a<L_{x}$, and $L_{x}=0$, then $a=0$ and $R_{q}=0$, which corresponds to the case without corrugation, exactly as if $L_{x}$ was infinite). Depending on the value of $L_{x}$, the peak of the transmission coefficient shifts towards either higher or lower angles in an irregular manner (the computational results show that a shift on the left occurred for $\mathrm{Lx}=7 \mathrm{~mm}$ and $9 \mathrm{~mm}$ and a shift on the right for $L_{x}=10 \mathrm{~mm}$ and $11 \mathrm{~mm}$ ) whereas the width is subjected to small variations. As can be seen, the increase of the $R_{q}$ caused by the variations of each of the parameters $h_{r}, a$ or $L_{x}$ does not necessary lead to similar evolutions of the transmission coefficients: in Figs. 4(a) and (b) the amplitude of the transmission coefficient diminishes monotonically with the considered parameter ( $h_{r}$ or $a$ ) whereas in Fig. 4(c) its amplitude together with its shift are irregularly depending on $L_{x}$.

\subsubsection{Keeping a constant $R_{q}$}

Fig. 4(d) shows the evolution of the transmission coefficient at constant $R_{q}$. The parameter $L_{x}$ is fixed and equal to $8 \mathrm{~mm}$ while $a$ and $h_{r}$ have opposite variations. In Fig. 4(d), the base $a$ of the triangle diminishes while at the same time its height $h_{r}$ increases: this amounts to considering the case of Section 3.2.2. devoted to the occupation by the triangle of the unit cell. The increasing of $h_{r}$ leads to a behavior of the peak similar to that observed in Fig. 4(a), i.e., a shift of the initial position of the maximum toward small angles.

The conclusion to be drawn from the analysis of Section 3.2 is that the increase of the height of the corrugation is accompanied by a diminishing of the amplitude of the transmission coefficient and a shift of the maximum toward smaller resonance angles. This
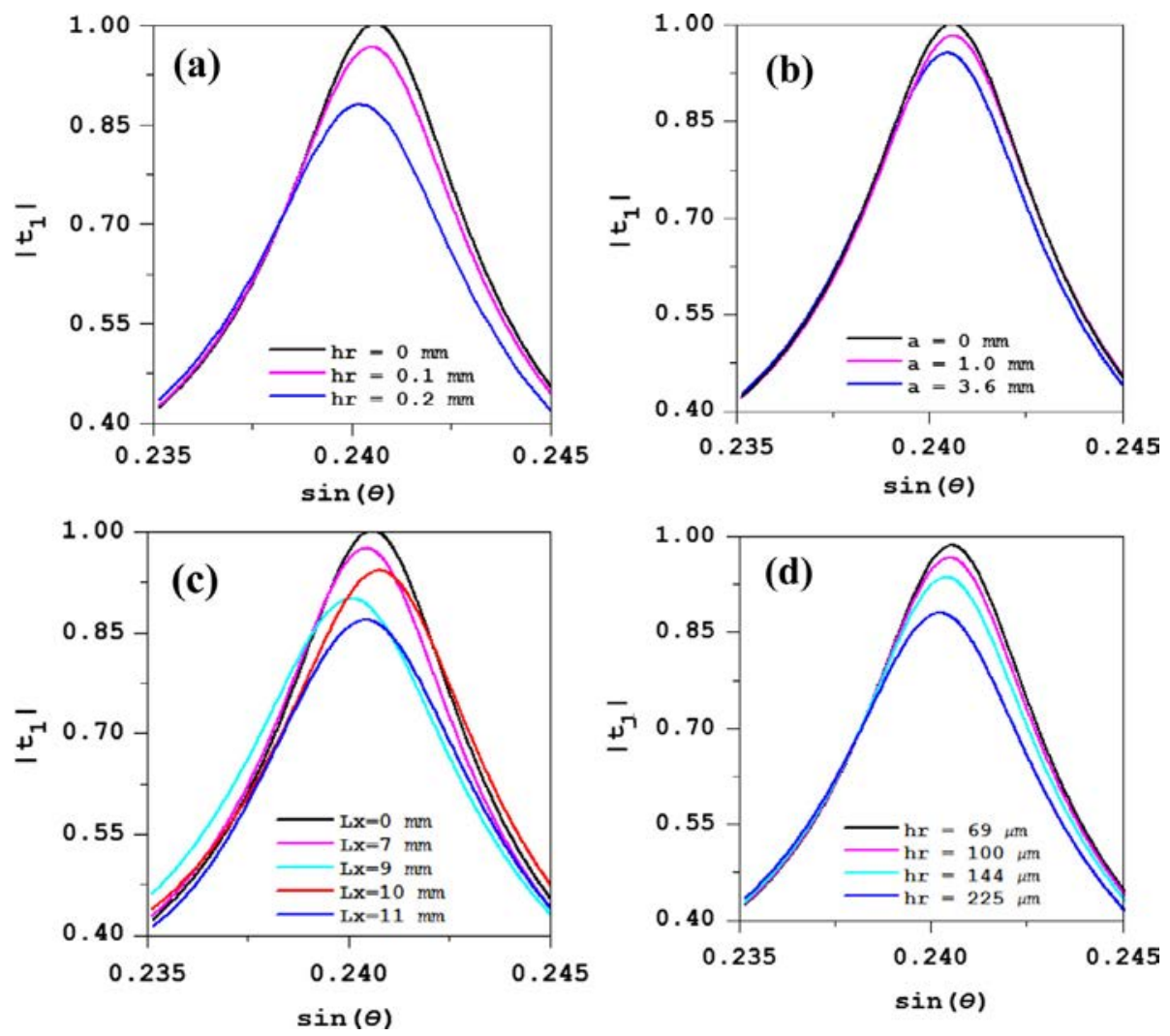

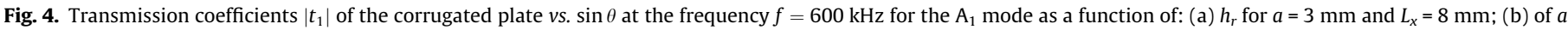
for $h_{r}=0.12 \mathrm{~mm}$ and $L x=8 \mathrm{~mm}$; (c) of $L_{x}$ for $a=3 \mathrm{~mm}$ and $h_{r}=0.12 \mathrm{~mm}$ (for the flat plate); (d) of $h_{r}$ and $a$ with $L_{X}$ fixed for preserving a constant $R_{q}$. 
seems rather logical concerning the amplitude, since the corrugation at the insonated face increases the amount of scattered energy.

\section{Comparisons between rheological and FEM models. Discussions}

The next objective is to show that the two models, one based on the simulation of a real corrugated interface and the other one based on the use of a simple rheological model lead to comparable results. In order to obtain a link between the two approaches, it is interesting to quantitatively compare the evolution of the shape of the resonance width with $h_{r}$ by FEM and the evolution of the imaginary part of the wave number with the complex stiffness $K_{L}$ (rheological model).

Using the FEM model results, the determination of the width of the resonance and of the angle of excitation is done by the least squares fitting procedure, in the vicinity of the selected maximum. By restricting the domain where the identification is carried out, the hypothesis of the isolated resonance is respected. The correlation coefficient between the computed data and the values of $T$ obtained from Eq. (17) is at least 0.99 for all the computations.

The resonances of the $A_{1}$ and $S_{1}$ modes have been characterized for five values of the height $h_{r}$. For the A1 mode (S1 mode, respectively), the values of the wavenumber $\bar{k}_{x, p, a}^{\prime}$ and of the width $\tilde{\Gamma}_{p, a}$ $\left(\bar{k}_{x, p, s}^{\prime}\right.$ and $\tilde{\Gamma}_{p, s}$, respectively) are plotted versus $h_{r}$. In order to decide which of the real part or of the imaginary part is more sensitive to the corrugation height, it seemed relevant to compute the relative values $\eta_{\theta}$ and $\eta_{\gamma}$ given by Eqs. (18) and (19). Results from FEM simulations are presented on Fig. 5(a) and (b). Next, the stiffness $K_{L}^{\prime}$ and $\beta$ can be estimated straightforwardly for each mode from the values of $\eta_{\theta}$ and $\eta_{\gamma}$ just obtained by using the following formulas derived from Eqs. (18) and (19):

$K_{L}^{\prime}=-\frac{\gamma_{p, a, s} K_{L 0, p, a, s}}{4 \eta_{\theta} \bar{k}_{x, p, a, S}}$

and

$\beta=-\frac{2 \eta_{\gamma} K_{L}^{\prime}}{K_{L 0, p, a, s}}$
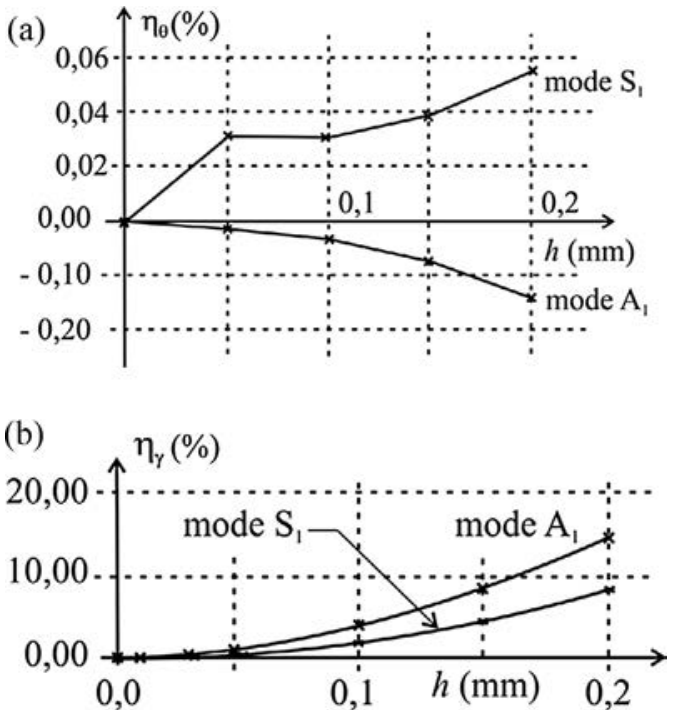

Fig. 5. Relative variations: (a) of $\eta_{\theta}$ and (b) of $\eta_{\gamma} v s$. $h_{r}$ for $\mathrm{A}_{1}$ and $\mathrm{S}_{1}$ mode at $600 \mathrm{kHz}$ $\left(a=3 \mathrm{~mm}, L_{x}=8 \mathrm{~mm}\right)$.
Values corresponding to the case of the $A_{1}$ mode, are consigned in Table 1 . The stiffness decreases when the height of the corrugation $h_{r}$ increases and its order of magnitude agrees well with our finding that for $K_{L}>10^{14} \mathrm{Nm}^{-3}$, the complex solutions of the dispersion equation are the same as those of a flat immersed plate with classical boundary conditions at each face given by Eq. (7).

On Fig. 6(a) is shown the continuous variation of the computed stiffness, whereas on Fig. 6(b) the coefficient $\beta$ is plotted for a wide range of the roughness parameters $R_{q}$.

It is remarkable that for small corrugation heights $(<50 \mu \mathrm{m})$ which are more difficult to measure, the stiffness $K_{L}^{\prime}$ has an almost linear variation, simplifying the correlation with the roughness $R_{q}$ values. The $\beta$ coefficient which depends on $K_{L}^{\prime}$, is almost linearly decreasing with increasing $R_{q}$ for even larger ranges of the corrugation heights.

However, the relation between the two quantities is not linear ( $\beta$ is proportional to $\left(K_{L}^{\prime}\right)^{2}$ and they tend asymptotically to a constant value as the $R_{q}$ roughness parameter increases.

We made the comparisons for two heights $\left(h_{r}=0.1 \mathrm{~mm}\right.$ and $0.2 \mathrm{~mm}$, see Fig. 4(a)). The results are shown in Fig. 7. The solid lines represent the FEM simulations, the solid line with circles or squares the Breit-Wigner approximations.

A better fit is obtained for $h_{r}=0.1 \mathrm{~mm}$ than for $h_{r}=0.2 \mathrm{~mm}$. In this last case, both the height and the half width of the peak of the Breit-Wigner curve disagree with the FEM model but only in a very limited extent. The factors behind these discrepancies can be the order of approximation at which the Breit-Wigner terms were expressed. Nevertheless, at the order of the approximations considered here, it can be observed that for the rheological model, as well as for the numerical simulations, the peaks shift more and more to lower frequencies when $h_{r}$ increases.

\section{Conclusions}

The results provided by FEM simulations and by a rheological model have been compared for transmission coefficient of the $A_{1}$ Lamb mode of a corrugated plate immersed in water. The roughness studied here is made of periodically spaced identical small triangles on one face of the plate. The choice of this mode and the test frequency is motivated by the optimal results, obtainable for any plate. We showed that by fitting these two approaches, it becomes possible to predict the effect of the roughness, by using a simple spring interface model, but with a complex valued stiffness. The approach investigated here shows, as in previous works [22,26], that it is the variation of the resonance widths, which indicate clearly the presence of surface corrugations and quantitative information about these corrugations.

For an increasing roughness, a shift of the resonance peak is observed in the simulations and in the reported rheological model used in the paper. The advantage of the present model which proposes a complex valued stiffness for the solid-liquid interface is the capacity to include the simultaneous influence of two geometrical parameters which are correctly describing the corrugation. From the determined parameters, the roughness $R_{q}$ parameter can be obtained. Moreover, in practical applications using a narrow ultrasonic beam, information on the immersed surface local imperfections, can thus be obtained faster than by using a profilometer.

\section{Appendix A.}

Let the index $a$ ( $s$, resp.) indicate the quantity related to the antisymmetric (symmetric, resp.) vibrations of the flat plate. The reflection coefficients $r_{1}$ (at the rough interface) and $r_{2}$ (at the flat interface) together with the transmission coefficient $t_{1}$ by the 
Table 1

Values of the stiffness constant $K_{L}^{\prime}$ and of the parameter $\beta$ for the $\mathrm{A}_{1}$ mode.

\begin{tabular}{lllll}
\hline$h_{r}(\mathrm{~mm})$ & $\eta_{\theta}(\%)$ & $\eta_{\gamma}(\%)$ & $K_{L}^{\prime}\left(\mathrm{Nm}^{-3}\right)$ & $\beta$ \\
\hline 0.05 & -0.02 & 0.05 & $1.357 \times 10^{14}$ & -0.466 \\
0.1 & -0.04 & 4 & $6.788 \times 10^{13}$ & -0.932 \\
0.15 & -0.08 & 8 & $3.394 \times 10^{13}$ & -0.932 \\
0.2 & -0.15 & 15 & $1.810 \times 10^{13}$ & -0.932 \\
\hline
\end{tabular}

(a) $1,8 \mathrm{E}+14$

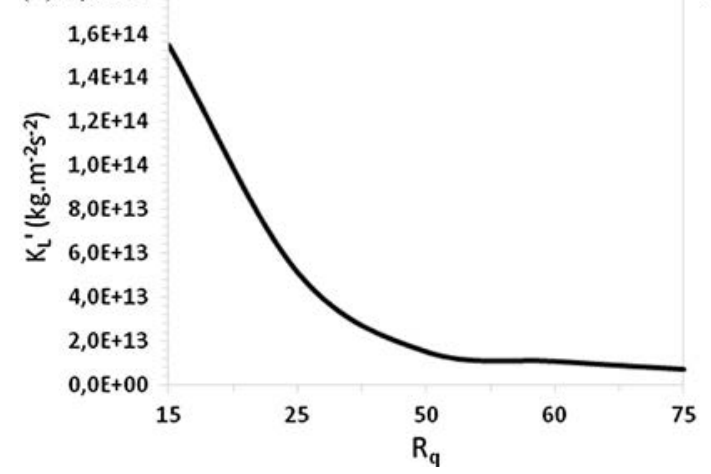

(b)

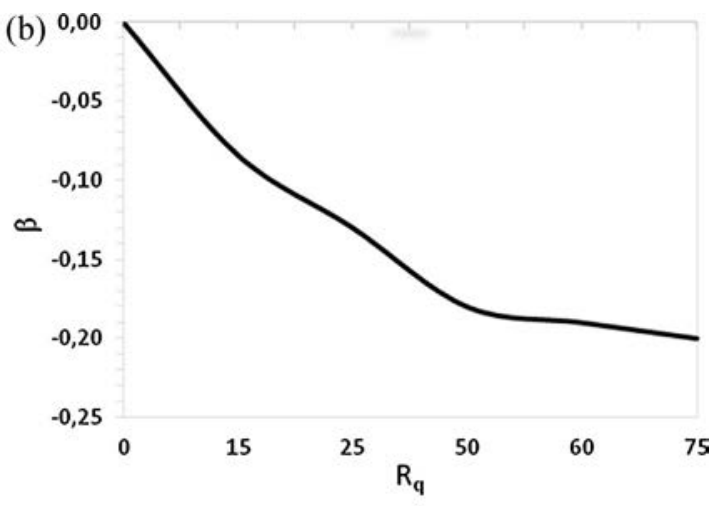

Fig. 6. The stiffness $K_{L}^{\prime}$ (a) and coefficient $\beta$ (b) for a wide range of roughness parameters $R_{q}$.

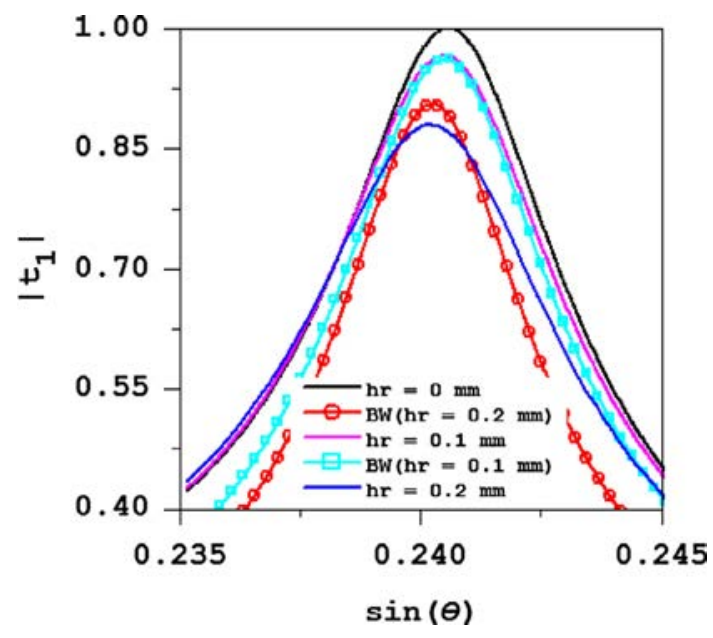

Fig. 7. Comparison of the FEM results (solid line) with the Breit-Wigner approximations computed by using the data of Table 1 (solid line with circles or squares).

plate, on account of the boundary conditions applied to identical fluid on the two faces, Eqs. (6) and (7), can be written

$r_{1}=\frac{\boldsymbol{C}^{+}}{\boldsymbol{C}^{-}} \times \frac{\left(C_{a}-i \tau^{+}\right)\left(C_{s}-i \tau\right)+\left(C_{a}+i \tau\right)\left(C_{s}+i \tau^{+}\right)}{\left(C_{a}+i \tau^{-}\right)\left(C_{s}-i \tau\right)+\left(C_{a}+i \tau\right)\left(C_{s}-i \tau^{-}\right)}$

$r_{2}=\frac{\left(C_{a}-i \tau^{-}\right)\left(C_{s}-i \tau\right)+\left(C_{a}+i \tau\right)\left(C_{s}+i \tau^{-}\right)}{\left(C_{a}+i \tau^{-}\right)\left(C_{s}-i \tau\right)+\left(C_{a}+i \tau\right)\left(C_{s}-i \tau^{-}\right)}$

$t_{1}=\frac{1}{\boldsymbol{C}^{-}} \times \frac{2 i \tau\left(C_{a}+C_{s}\right)}{\left(C_{a}+i \tau^{-}\right)\left(C_{s}-i \tau\right)+\left(C_{a}+i \tau\right)\left(C_{s}-i \tau^{-}\right)}$,

where

$\boldsymbol{C}^{ \pm}=1 \pm i \frac{K_{L 0}}{K_{L}}, \quad K_{L 0}=\frac{\rho_{1} c_{1} \omega}{\bar{k}_{z 1}}$, $\tau=\frac{\rho_{1}}{\rho_{s}} n_{T}^{4} \frac{\bar{k}_{z L}}{\bar{k}_{z 1}}, \quad \tau^{ \pm}=\frac{\tau}{\boldsymbol{C}^{ \pm}}$

$C_{a}=\left(2 \bar{k}_{x}^{2}-n_{T}^{2}\right)^{2} \tan \left(\frac{\bar{k}_{z L} k_{1} h}{2}\right)+4 \bar{k}_{x}^{2} \bar{k}_{z L} \bar{k}_{z T} \tan \left(\frac{\bar{k}_{z T} k_{1} h}{2}\right)$,

and

$C_{s}=\left(2 \bar{k}_{x}^{2}-n_{T}^{2}\right)^{2} \cot \left(\frac{\bar{k}_{z L} k_{1} h}{2}\right)+4 \bar{k}_{x}^{2} \bar{k}_{z L} \bar{k}_{z T} \cot \left(\frac{\bar{k}_{z T} k_{1} h}{2}\right)$.

with

$\bar{k}_{x}=\sin \theta, \bar{k}_{z 1}=\left(1-k_{x}^{2}\right)^{1 / 2}>0$ for $0 \leqslant \theta<90^{\circ}$, and $n_{L, T}$

$=\frac{c_{1}}{c_{L, T}}$.

Since $n_{L}<n_{T}<1$, and since emphasis is on angular domain I where $\bar{k}_{x}<n_{L}$, we have $\bar{k}_{z L, T}=\left(n_{L, T}^{2}-\bar{k}_{x}^{2}\right)^{1 / 2}>0$ : both $L$ and $T$ waves propagate.

\section{References}

[1] D. Whitehouse, Surfaces and Their Measurement, Taylor Hobson, London, 2002.

[2] J.W.S. Rayleigh, Theory of Sound, vol. 2, Dover Publications, New York, 1945.

[3] J.W. Miles, On nonspecular reflection at a rough surface, J. Acoust. Soc. Am. 26 (12) (1954) 191-199.

[4] M. Biot, Reflection on a rough surface from an acoustic point source, J. Acoust. Soc. Am. 29 (111) (1957) 1193-1200.

[5] M. Biot, On the reflection of acoustic waves on a rough surface, J. Acoust. Soc. Am. 30 (15) (1958) 479-480.

[6] R. Sinclair, Velocity dispersion of Rayleigh waves propagating along rough surfaces, J. Acoust. Soc. Am. 50 (13) (1970) 841-845.

[7] G. Quentin, M. de Billy, F. Cohen Tenoudji, J. Doucet, A. Jungman, et al., Experimental results on the scattering of ultrasound by randomly or periodically rough surfaces, in: IEEE Ultrasonics Symposium Proceedings, 1975.

[8] S.-L. Chuang, R.K. Johnson, et al., Acoustic wave scattering from a fluid/solid periodic rough surface, J. Acoust. Soc. Am. 71 (16) (1982) 1368-1376.

[9] K. Mampaert, O. Leroy, et al., Reflection and transmission of normally incident ultrasonic waves on periodic solid-liquid interfaces, J. Acoust. Soc. Am. 83 (14) (1988) 1390-1398. 
[10] A. Wirgin, «Reflection from a corrugated surface, J. Acoust. Soc. Am. 68 (12) (1980) 692-699.

[11] N. Haines, D. Langston, et al., The reflection of ultrasonic pulses from surfaces, J. Acoust. Soc. Am. 67 (15) (1980) 1443-1454.

[12] J.A. Ogilvy, Theory of Wave Scattering From Random Rough Surfaces, I.O.P, Bristol, 1991.

[13] T.M. Elfouhaily, C.-A. Guérin, et al., A critical survey of approximate scattering wave theories from random rough surfaces, Waves Random Media 14 (2004) $1-40$.

[14] R. Fiorito, W. Madigosky, H. Überall, et al., Resonance theory of acoustic waves interacting with an elastic plate, J. Acoust. Soc. Am. 66 (16) (1979) 1857-1866.

[15] G. Maze, J.-L. Izbicki, J. Ripoche, et al., Resonances of plates and cylinders: guided waves, J. Acoust. Soc. Am. 77 (14) (1985) 1352-1357.

[16] A. Pilarski, J.L. Rose, K. Balasubramaniam, et al., The angular and frequency characteristics of reflectivity from a solid layer embedded between two solids with imperfect boundary conditions, J. Acoust. Soc. Am. 87 (12) (1990) 532542.

[17] V. Vlasie, M. Rousseau, et al., Acoustical validation of the rheological models for a structural bond," Wave Motion 37 (13) (2003) 333-349.

[18] P.B. Nagy, L. Adler, Attenuation of reflected and transmitted ultrasonic waves through a rough interface, in: Ultrasonics Symposium, Denver, 1987.

[19] P.B. Nagy, L. Adler, et al., Surface roughness induced attenuation of reflected and transmitted ultrasonic waves, J. Acoust. Soc. Am. 82 (11) (1987) 193-197.
[20] J. Duclos, J.-L. Izbicki, O. Lenoir, J.M. Conoir, et al., Resonant formalism for the liquid-solid interface Rayleigh mode, Acta Acoust. 2 (1994) 375-378.

[21] B.W. Drinkwater, R.S. Dwyer-Joice, P. Cawley, et al., A study of the interaction between ultrasound and partially contacting solid-solid interface, Proc. Roy. Soc. Lond. 452 (1996) 2613-2628.

[22] D.E. Chimenti, O.I. Lobkis, et al., The effect of rough surfaces on guided waves in plates, Ultrasonics 36 (1998) 155-162.

[23] D. Leduc, A.-C. Hladky, B. Morvan, J.-L. Izbicki, P. Pareige, et al., Propagation of Lamb waves in a plate with a periodic grating: Interpretation by phonon, J. Acoust. Soc. Am. 118 (14) (2005) 2234-2239.

[24] J.P. Jones, J.S. Whittier, et al., Waves at a flexibly bonded interface, J. Appl. Mech. 34 (14) (1967) 905-909.

[25] O.I. Lobkis, D.E. Chimenti, et al., Elastic guided waves in plates with surface roughness. I. Model calculation, J. Acoust. Soc. Am. 102 (11) (1997) 143-149.

[26] C. Potel, D. Leduc, B. Morvan, C. Depollier, A.-C. Hladky-Hennion, J.-L. Izbicki, P. Pareige, M. Bruneau, et al., Lamb wave attenuation in a rough plate. I. Analytical and experimental results in an anisotropic plate, J. Appl. Phys. 104 (2008) 074908.

[27] COMSOL Multiphysics User Manual, COMSOL AB, 2014. <http:// www.comsol.com/comsol-multiphysics> (accesed 0802 2015).

[28] F. Nataf, Absorbing boundary conditions and perfectly matched layers in wave propagation problems, Radon Ser. Comput. Appl. Math. 14 (2013) 19-231. 